Background Pemetrexed is an expensive oncological drug, used in combination with platinum derivatives (cisplatin/carboplatin) in the first line treatment of unresectable malignant pleural mesothelioma. In Italy, this indication is no longer subject to web-based monitoring (Onco-AIFA Register) to ensure its use appropriateness. Purpose To assess the effectiveness in patients treated at the Istituto Oncologico Veneto (IOV) compared to the pivotal trial.

Materials and Methods This observational analysis was performed on all patients with pleural mesothelioma treated at the IOV from 01/12/2006 to 30/04/2011; the data were extracted from both paper and computerised medical records. The median Overall Survival (OS) and Time To Progression (TTP) were calculated as updated on 31/05/2012 according to the intention to treat.

Results All 46 patients (32 males and 14 females) were evaluated in terms of OS. TTP was calculated only for the 41 evaluable patients ( 29 males and 12 females); 5 patients lost owing to lack of information at follow-up.

The median OS/TTP values were respectively 14.2/8.9 months (vs. pivotal trial 12.1/5.7 months).

The majority of patients received the less toxic protocol pemetrexed + carboplatin, which contributed to the better OS/TTP. Better OS/TTP might be related to the use in a neoadjuvant regimen (16 patients: 10 males and 6 females); a specific stratified analysis showed TTP/OS median of 27.8/18.6 months.

Conclusions To confirm the better effectiveness of the carboplatin + pemetrexed protocol, further data on a greater number of patients, neoadjuvant treatment, treatment toxicity and patient performance status are needed.

Since the effectiveness of this high-cost oncological drug is not monitored at the national level, local monitoring is required to ensure appropriateness.

The computerised medical record is a pre-requisite for protocol standardisation and a tool of information standardisation/updating.

This work represents an easy, versatile methodological model with significant health implications.

A widely shared computerised medical record is a powerful tool for epidemiological investigations; an established network allowing benchmarking is a valid and independent decision-making tool.

No conflict of interest.

\section{DGI-033 EVALUATION OF CRIZOTINIB TREATMENT IN PATIENTS WITH NON-SMALL CELL LUNG CANCER}

doi:10.1136/ejhpharm-2013-000276.299

AM Villalba-Moreno, T Trinidad-Desongles, MA Pérez-Moreno, HL Acosta-García, J Cotrina-Luque, E Chamorro-de Vega, M Galván-Banqueri. Hospital Universitario Virgen del Rocío, Pharmacy, Seville, Spain

Background Crizotinib is a cytostatic oral ALK inhibitor, a newlyintroduced oral cytostatic to treat non-small cell lung cancer (NSCLC) that has been accessible through an expanded use programme prior to marketing authorization.

Purpose To analyse the effectiveness and safety of crizotinib treatment in patients with NSCLC in a tertiary hospital.

Materials and Methods A retrospective descriptive study of patients taking crizotinib from August 2011 to July 2012. The following information was collected: demographic (gender and age), background (smoker/non-smoker), basal situation (Performance Status (PS), ALK-positive or negative), diagnosis and staging, dose of crizotinib, results (progress and current status) and adverse reactions. The average length of survival was determined using SPSS 20. The information sources were the electronic health records.

Results 4 patients were recruited. 3 (75\%) were women. The mean age was 47. All the patients were non-smokers. Initial situation: 3 patients had a PS of 1 and the other one had 2. All of them were
ALK-positive and were diagnosed with stage IV NSCLC. 2 patients received crizotinib $250 \mathrm{mg} / 12 \mathrm{~h}$ and the other $2200 \mathrm{mg} / 12 \mathrm{~h}$. Evolution: in $2(50 \%)$ patients the tumour mass in the lungs did not change. In $1(25 \%)$ the lung tumour shrank slightly. To sum up: $3(75 \%)$ patients presented stable disease and 1 died. Adverse reactions: $3(75 \%)$ patients had gastrointestinal reactions (diarrhoea and mucositis), 2 (50\%) patients presented asthenia and 1 (25\%) visual disturbances. Lastly, the average length of survival was 6 months (IC95\%, 2.33-9.66).

Conclusions Due to the low number of patients recruited the effectiveness of the treatment cannot be demonstrated. Nevertheless, it is important to highlight that the disease stabilised in 3 out of 4 patients. Gastrointestinal problems were the most frequent adverse reactions. It is important to detect ophthalmological adverse reactions in time to begin patient tracking. This treatment is well tolerated in patients with a bad prognosis and few treatment options.

No conflict of interest.

\section{DGI-034 EVALUATION OF THE EFFICACY AND SAFETY OF MIFAMURTIDE IN OSTEOGENIC SARCOMA TREATMENT IN PAEDIATRIC PATIENTS}

doi:10.1136/ejhpharm-2013-000276.300

'S Berisa, 'E Lacalle, 'ME Carrasco, 'F Marcotegui, ${ }^{2} S$ Martínez, 'M Gutiérrez, 'M Castresana, 'M Elviro, 'E Pellejero. 'Hospital B Complejo Hospitalario de Navarra, Hospital Pharmacy, Pamplona, Spain; ${ }^{2}$ Hospital San Pedro, Hospital Pharmacy, Logroño, Spain

Background Osteosarcoma is a relatively common bone tumour; with an incidence of 0.2 to $3 / 100000$, it is an orphan disease. Mifamurtide has managed to increase survival without increasing side effects.

Purpose To evaluate the safety and efficacy of mifamurtide in two paediatric patients diagnosed with osteogenic sarcoma.

Materials and Methods We conducted a prospective study of two paediatric patients diagnosed with osteogenic sarcoma. Weekly, we attended the oncology sessions and we tracked them during the chemotherapy, and after that, through the electronic clinical history.

Mifamurtide is indicated in children, adolescents and young adults for the treatment of high-grade resectable non-metastatic osteosarcoma after surgical resection. It is used in combination with post-operative chemotherapy.

In the two cases, the treatment followed the SEOP-SO-2010 guidelines of the Spanish Society of Paediatric Oncology for 37 weeks.

After surgery (week 15) mifamurtide was started as adjuvant treatment: $2 \mathrm{mg} / \mathrm{m}^{2}$ twice weekly for the first 12 weeks and followed by once-weekly for an additional 24 weeks, for a total of 48 infusions in 36 weeks.

Results Chemotherapy started according to protocol, the patients were aged 12 and 15 years (July and November 2010, respectively).

One patient had a flu-like reaction after the first dose of mifamurtide, so the following doses were administered with premedication (acetaminophen and dexchlorpheniramine). Other side effects: anaemia and thrombocytopenia, requiring human stimulating factors and platelet concentrates; vomiting was treated with aprepitant.

When chemotherapy finished, the patients were in complete remission, this situation continues today, 10 and 13 months later.

Conclusions The SEOP protocol plus mifamurtide achieved complete remission in both cases.

The use of mifamurtide can be considered safe and it did not increase side effects, we observed only a flu-like reaction attributed to mifamurtide which resolved with premedication. 